\title{
A comprehensive analysis of drug resistance molecular markers and Plasmodium falciparum genetic diversity in two malaria endemic sites in Mali
}

Seidina A. S. Diakité ${ }^{12^{*}} \mathbb{0}$, Karim Traoré1, Ibrahim Sanogo ${ }^{1}$, Taane G. Clark ${ }^{3}$, Susana Campino ${ }^{3}$, Modibo Sangaré1,

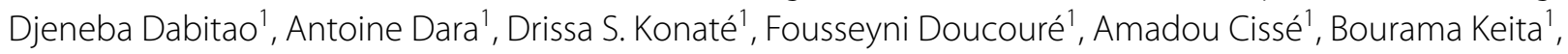
Mory Doumbouya', Merepen A. Guindo', Mahamoudou B. Toure', Nafomon Sogoba', Seydou Doumbia', Gordon A. Awandare ${ }^{2}$ and Mahamadou Diakité ${ }^{1}$

\begin{abstract}
Background: Drug resistance is one of the greatest challenges of malaria control programme in Mali. Recent advances in next-generation sequencing (NGS) technologies provide new and effective ways of tracking drug-resistant malaria parasites in Africa. The diversity and the prevalence of Plasmodium falciparum drug-resistance molecular markers were assessed in Dangassa and Nioro-du-Sahel in Mali, two sites with distinct malaria transmission patterns. Dangassa has an intense seasonal malaria transmission, whereas Nioro-du-Sahel has an unstable and short seasonal malaria transmission.
\end{abstract}

Methods: Up to 270 dried blood spot samples (214 in Dangassa and 56 in Nioro-du-Sahel) were collected from P. falciparum positive patients in 2016. Samples were analysed on the Agena MassARRAY ${ }^{\circledR}$ iPLEX platform. Specific codons were targeted in Pfcrt, Pfmdr1, Pfdhfr, and Pfdhps, Pfarps 10, Pfferredoxin, Pfexonuclease and Pfmdr2 genes. The Sanger's 101-SNPs-barcode method was used to assess the genetic diversity of $P$. falciparum and to determine the parasite species.

Results: The Pfcrt_76I chloroquine-resistance genotype was found at a rate of $64.4 \%$ in Dangassa and $45.2 \%$ in Nioro-du-Sahel $(p=0.025)$. The Pfdhfr_511-59R-108N pyrimethamine-resistance genotype was $14.1 \%$ and $19.6 \%$, respectively in Dangassa and Nioro-du-Sahel. Mutations in the Pfdhps_S436-A437-K540-A581-613A sulfadoxine-resistance gene was significantly more prevalent in Dangassa as compared to Nioro-du-Sahel $(p=0.035)$. Up to 17.8\% of the isolates from Dangassa vs 7\% from Nioro-du-Sahel harboured at least two codon substitutions in this haplotype. The amodiaquine-resistance Pfmdr1_N86Y mutation was identified in only three samples (two in Dangassa and one in Nioro-du-Sahel). The lumefantrine-reduced susceptibility Pfmdr1_Y184F mutation was found in 39.9\% and 48.2\% of samples in Dangassa and Nioro-du-Sahel, respectively. One piperaquine-resistance EXo_E415G mutation was found in Dangassa, while no artemisinin resistance genetic-background were identified. A high P. falciparum diversity was observed, but no clear genetic aggregation was found at either study sites. Higher multiplicity of infection was observed in Dangassa with both COIL $(p=0.04)$ and Real McCOIL $(p=0.02)$ methods relative to Nioro-du-Sahel.

\footnotetext{
*Correspondence: sdiakite@icermali.org

${ }^{1}$ Malaria Research and Training Center, University of Sciences, Technics

and Technologies of Bamako (USTTB), Bamako, Mali

Full list of author information is available at the end of the article
} 
Conclusions: This study reveals high prevalence of chloroquine and pyrimethamine-resistance markers as well as high codon substitution rate in the sulfadoxine-resistance gene. High genetic diversity of P. falciparum was observed. These observations suggest that the use of artemisinins is relevant in both Dangassa and Nioro-du-Sahel.

Keywords: Plasmodium falciparum, Drug-resistance, Molecular surveillance, Next-generation sequencing

\section{Background}

Despite numerous advances in malaria control strategies, the disease still kills countless children worldwide, mainly in sub-Saharan African countries. The World Health Organization (WHO) reported no significant progress in reducing global malaria burden during the period from 2015 to 2017 [1]. In Mali, as well as in the rest of the world, resistance to anti-malarial drugs is one of the greatest challenges of the National Malaria Control Programme (NMCP) [2, 3]. High prevalence of resistance to chloroquine and sulfadoxine-pyrimethamine (SP) led the Malian NMCP to switch to artemisinin- based combination therapy (ACT) in the 2000s, as recommended by the WHO [4]. Artemisinin and its derivative drugs (Artemisinins) are still very effective in Africa [5, 6]. Resistance to these drugs have been reported in East-Asia [7, 8]. One of the rationales of ACT use is to maintain long-term efficacy of artemisinins to P. falciparum [4]. To achieve this objective, the choice of the artemisinin partner drugs is important and should be made based on their effectiveness on circulating local parasite isolates. Consequently, it appears important to monitor the emergence and spread of the anti-malarial drug resistance in different geographical and endemic areas in order to inform the choice of the anti-malarial molecules to be associated with artemisinins in ACT $[9,10]$. Also, assessing the genetic diversity of $P$. falciparum populations in different regions may allow to tract malaria parasites circulating across different geographic areas.

Molecular markers of drug-resistance are very useful in identifying drug-resistant $P$. falciparum. They are used in epidemiological surveillance of drug-resistance including their emergence and spread monitoring. Molecular markers have been described for many of the common anti-malarial drug and are constituted of either single nucleotide substitution (SNP) or concatenated SNP in genes involved in parasite interaction with drugs. Evidence of association between several alleles in $P$. falciparum multi-drug resistance ( $P f m d r$ ) gene and chloroquine-resistance was reported in 1990 [11]. However, the K76T mutation in P. falciparum chloroquine resistance transporter gene (Pfcrt_K76T) mostly associated with the Pfcrt_72C-73V-74I-75E-76T haplotype in Africa is known as the strongest chloroquine-resistance marker in P. falciparum [12-15]. Resistance to pyrimethamine has also been associated with numbers of mutations combination in the $P$. falciparum dihydrofolate-reductase (Pfdhfr) gene, such as the Pfdhfr_51I-59R-164L [16] and Pfdhfr_51R-59N-108I genotypes [17, 18]. Also various combinations of substitutions at the codons 436, 437, 540, 581 and 613 of the P. falciparum dihydropteroate-synthetase (Pfdhps) gene may confer resistance to sulfadoxine [19]. The greater the number of substitution accumulation is in the gene, the more the parasite gets resistant to the sulfadoxine. The Pfdhps_437G-540E genotype was associated with substantially decreased susceptibility to sulfadoxine. In vitro experiments have shown that $\mathrm{N} 86 \mathrm{Y}$ mutation in the P. falciparum multidrug resistance-1 (Pfmdr1) gene (Pfmdr1_86Y) increases the fifty percent inhibitory concentrations (IC50) of chloroquine and amodiaquine $[20,21]$.

Mutations in multidrug resistance protein ( $p f m d r 1)$ have been associated with various parasite response to mefloquine and lumefantrine [22]. Reduced susceptibility to lumefantrine has been associated with the Pfmdr1_Y184F mutation [23-25]. In a recent genomewide association study (GWAS), the E415G mutation, a SNP in P. falciparum exonuclease gene (Pfexo_E415G)) was associated with ex vivo piperaquine IC50 of parasite isolates from Cambodia [26]. The co-inheritance of several mutations in P. falciparum Apicoplast Ribosomal Protein S10 (Pfarps10) gene [Pfarps10_127M-128Y/H], the P. falciparum ferredoxin (PfFd) gene [Pffd_ 193Y], the PfCRT gene: [Pfcrt_326S-356T], and the PfMDR2 [Pfmdr2_T484I] were shown to constitute a genetic background that allows for the emergence of mutations in the $P$. falciparum kelch 13 gene [27], a known artemisinin resistance locus [28-30]. This haplotype is referred to as artemisinin-resistant parasite genetic background (Artresistant PGB).

All thought previous studies has provided insights into some anti-malarial drug resistance markers in the south of Mali [31, 32], the risk of $P$. falciparum infection is variable from south (with intense and long transmission) to north (with unstable and short transmission) in Mali. This variability is related to environmental and climatic conditions. The level of transmission may also impact the malaria parasite genetic diversity and the spread of resistance to anti-malarial drugs. In fact, the higher the malaria transmission is, the more diverse the parasite population is, the more likely the parasite gets resistant to anti-malarial drugs. 
In this study, both the genetic diversity of $P$. falciparum and the distribution of drug resistance markers were assessed in two distinct eco-climatic areas in Mali.

\section{Methods \\ Study sites}

The epidemiology, entomology and the impact of malaria vary widely across the Malian territory [33, 34]. The study was carried out in Dangassa (Lat $=12.150,925$, Long $=-8.206974)$ and Nioro-duSahel (Lat $=15.224674$, Long $=-9.583888)$. Dangassa is located in south savannah grassland of Mali along the right side of Niger River and has year-round access to water (Niger River). Malaria transmission is seasonal and mainly occurs from June to December. However, dry season transmission may occurs because of the Niger River. Indeed, mosquito breeding occurs primarily in microhabitats, such as the footprints of cattle and the receding river in the dry season, which leaves natural pools as well as man-made pools from river gold mining. Niorodu-Sahel is located in Sahelian region of Mali near the Islamic Republic of Mauritania. Malaria transmission is unstable and occurs from July to September. Anopheles gambiae breeding sites are strictly rain-dependent. Anopheles gambiae (about 95\%) and Anopheles arabiensis (about 5\%) are the predominant malaria vectors [35] in the two sites. Population migration occurs in the two localities mainly for gold mining for Dangassa and for herding or for trading for Nioro-du-Sahel. In both localities, malaria control strategies rely on the use of longlasting insecticide-impregnated nets (LLINs), ACT for treatment and sulfadoxine-pyrimethamine (SP) for intermittent preventive treatment of pregnant women (IPTp). In addition, the population may have access to other malaria drugs in the local market in the two localities.

\section{Study design and sample collection}

The study has been set up by the research team in collaboration with the local health staff for the entire malaria transmission season (June to December 2016). A medical team was installed at the health centre of the village. Population of all age group was encouraged to attend the local health centre if febrile, where they were examined by a study physician. Malaria cases were diagnosed by a rapid diagnostic test (RDT) [CareStart ${ }^{\mathrm{TM}}$ : Malaria HRP2/ pLDH(Pf/PAN) Combo] and/or microscopy. A four (4) $\mathrm{ml}$ venous blood was obtained from each TDR positive patient. A dried blood spot (DBS) was made by dropping approximatively $50 \mu \mathrm{l}$ of the venous blood on a filter paper. The DNA was extract from the DBS and used for $P$. falciparum genomic and host genetic studies. Confirmed malaria cases were classified as mild or severe according to the World Health Organization (WHO) definitions
[36], and treated according to the Malian NMCP guidelines (oral ACT for uncomplicated malaria, intramuscular (i.m) administration of artemether and/or quinine for severe malaria).

\section{DNA extraction, sample processing, and genotyping}

The dried blood spots on filter paper were sent to the Wellcome Sanger Institute for processing. DNA extraction was carried out using the Qiagen DNA Investigator Kit (No. 56504, Qiagen, Crawley, UK) for high-throughput robotic processing. DNA was eluted in $100 \mu \mathrm{l}$ TE buffer and stored at $-20{ }^{\circ} \mathrm{C}$ for later use. Extracted DNA underwent whole genome amplification (WGA) by primer-extension pre-amplification [37] or selective WGA [38] prior to genotyping. Genotyping was performed according to manufacturer's instruction on the Agena MassARRAY ${ }^{\circledR}$ iPLEX platform (Agena Bioscience, Hamburg, Germany). This system is able to accurately genotype large numbers of samples for multiple SNPs simultaneously. It previously been used in P. falciparum for sequencing validation of novel SNPs [39]. All genotypes were called from background adjusted peak intensities, which were normalized and called by batch. In brief, batches underwent calling using a heuristic algorithm which identifies intensity ranges for each SNP in single infection samples, and called mixed base loci ("heterozygous") based on those range thresholds, adjusting for background intensity. Batches were plate based and contained between 96 and 384 samples. These are necessary to generate per assay ranges of intensities to calculate background intensity levels.

\section{Parasite diversity and multiple infection assessment}

The malaria parasite diversity and multiplicity of infection were assessed using the Sanger's $P$. falciparum barcodes formed by concatenated genotypes at 101 SNPs across $P$. falciparum genome. These SNPs are all bi-allelic, at low to medium frequency in global parasite populations, and were chosen for their usefulness in analyses of relationship between $P$. falciparum parasites. They have been used previously to estimate levels of relationship and importation [40] as well as complexity of infection. Multiplicity of infection estimates were produced by both the programmes COIL [41] using default parameters and The Real McCOIL [42] using the bi-allelic SNP barcodes.

\section{Drug resistance markers assessment}

Specific mutations in $P$. falciparum genome were screened using the Agena MassARRAY ${ }^{\circledR}$ iPLEX platform as above. The following codons in the P. falciparum genome were screened for substitution: K76, N326, I356 in the Pfcrt gene, the N86, Y184, D1246 in the Pfmdr1 gene, the N51, C59, S108, I164 in the Pfdhfr gene, the 
S436, A337, K540, A581, A613 in Pfdhps V127, D128 in the Pfarps10 gene, the D193 in Pf-ferredoxin gene, the E415 in Pf-exonuclease gene, and T484 in Pfmdr2. Single isolate mutations or combination of mutations in these codons are associated with resistance to anti-malarial drugs as specified in the background section.

\section{Statistical analysis}

This was an inclusive study. All the confirmed malaria case detected during the study period in the two sites were enrolled. A Chi square test was used to compare the prevalence of molecular markers in the two study sites. When the effective $\geq 30$, the Z-test was considered. The significant $P$ value was set as equal or inferior to 0.05 .

For the Real McCOIL determination, the categorical method was used with the following parameters, $\operatorname{maxCOI}=25$, threshold_ind $=20$, threshold_site $=20$, totalrun $=10,000$, burnin $=1000, \quad \mathrm{M} 0=15, \quad \mathrm{e} 1=0.05$, $\mathrm{e} 2=0.05$, err_method $=1$. Principal component analysis was performed to assess and compare the parasite diversity between the two study sites.

\section{Results}

In total, 270 P. falciparum samples (214 from Dangassa and 56 from Nioro-du-Sahel) were analysed. The analysis of anti-malarial drug genes showed very high rates of mutations. The chloroquine-resistance Pfcrt-76 $\underline{T}$ genotype was significantly more prevalent in Dangassa $[64.4 \%(45.9 \% 76 \mathrm{~T}+18.4 \% 76 \mathrm{~K} / 76 \mathrm{~T})]$ compared to
Nioro-du-Sahel [45.2\% (37.7\% $76 \mathrm{~T}+7.5 \% \quad 76 \mathrm{~K} / 76 \mathrm{~T})]$ $(\mathrm{p}=0.025)$ (Fig. 1). This study revealed a very high but similar rate of codon-substitution in the pyrimethamine-resistance $P f d h f r$ gene at positions 51, 59, 108 and 164 in both Dangassa and Nioro-du-Sahel (Fig. 2) $(\mathrm{p}=0.2$ ). Respectively, $88.3 \%$ and $80 \%$ of the isolates from Dangassa and Nioro showed at least one substitution at these codons. However the prevalence of the particular pyrimethamine-resistance Pfdhfr_51I-19R$108 N$ genotype reached $14.1 \%$ and $19.6 \%$ in Dangassa and Nioro-du-Sahel, respectively. Higher rate of codon substitution was observed in the sulfadoxine-resistance Pfdhps gene at positions 436, 437, 540, 581 and 613. These substitutions were more prevalent in Dangassa as compared to Nioro-du-Sahel ( $p$ value $=0.035$ ). Up to $17.8 \%$ of the isolates from Dangassa against $7 \%$ for the isolates from Nioro-du-Sahel accumulated at least two (2) codon-substitutions were detected at these codons (Fig. 3).

The particular sulfadoxine-resistance haplotype Pfdhps_437G-540E was found in four isolates from Dangassa out of which only one exhibits the pyrimethamineresistance $P f d h f r \_51 I-19 R-108 N$ genotype (the quintuple Pfdhps_437G-540E/Pfdhfr_51I-19R-108N substitution).

The amodiaquine-resistance $P f m d r 1-\mathrm{N} 86 \mathrm{Y}$ mutation was identified in $14.9 \%$ (6\% Pfmdr1-Y86+8.8\% Pfmdr1-N86/Y86) and 14.2\% (8.9\% Pfmdr1-Y86+8.3\% Pfmdr1-N86/Y86) of the samples from Dangassa and Nioro-du-Sahel, respectively.

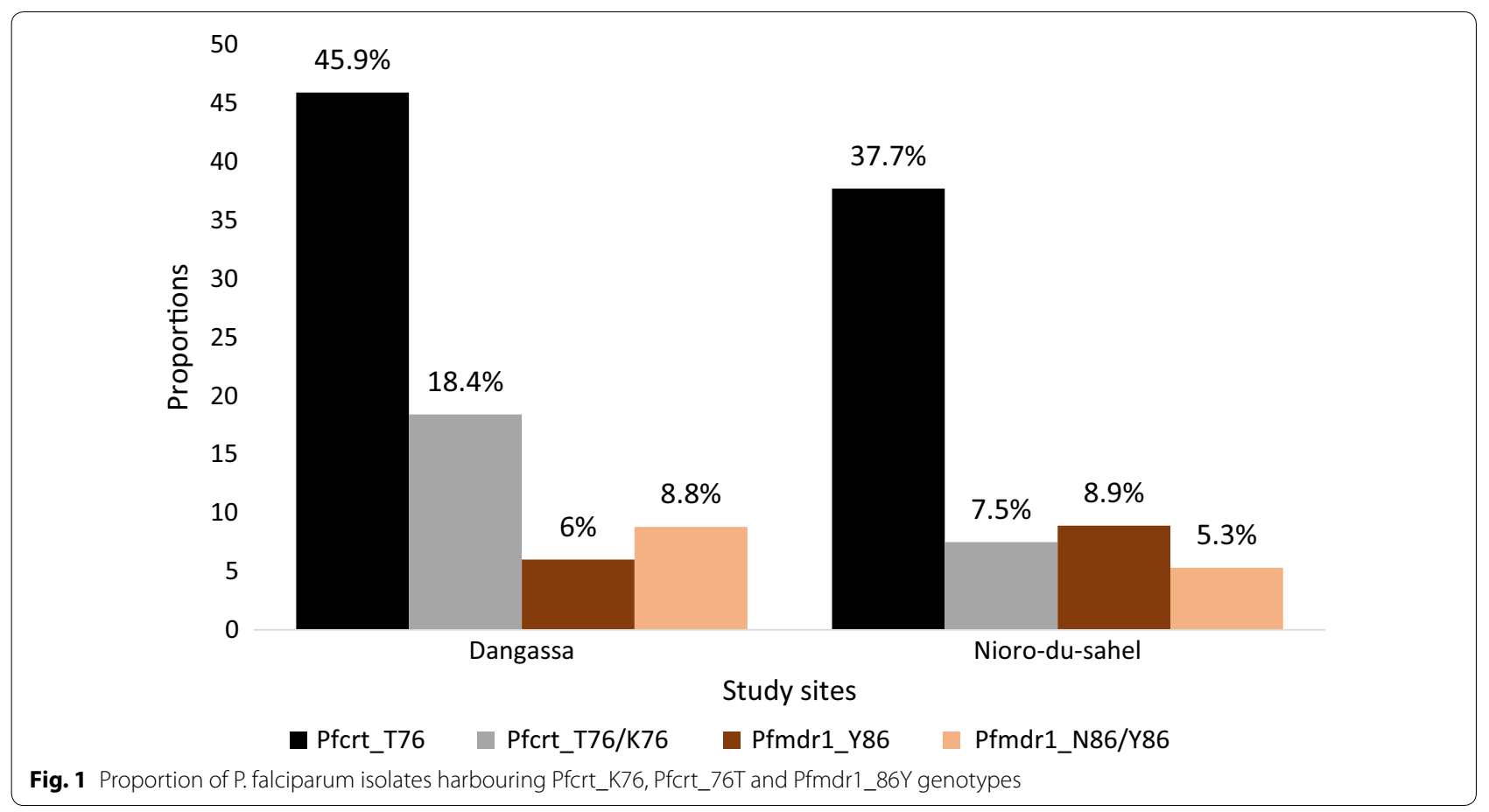




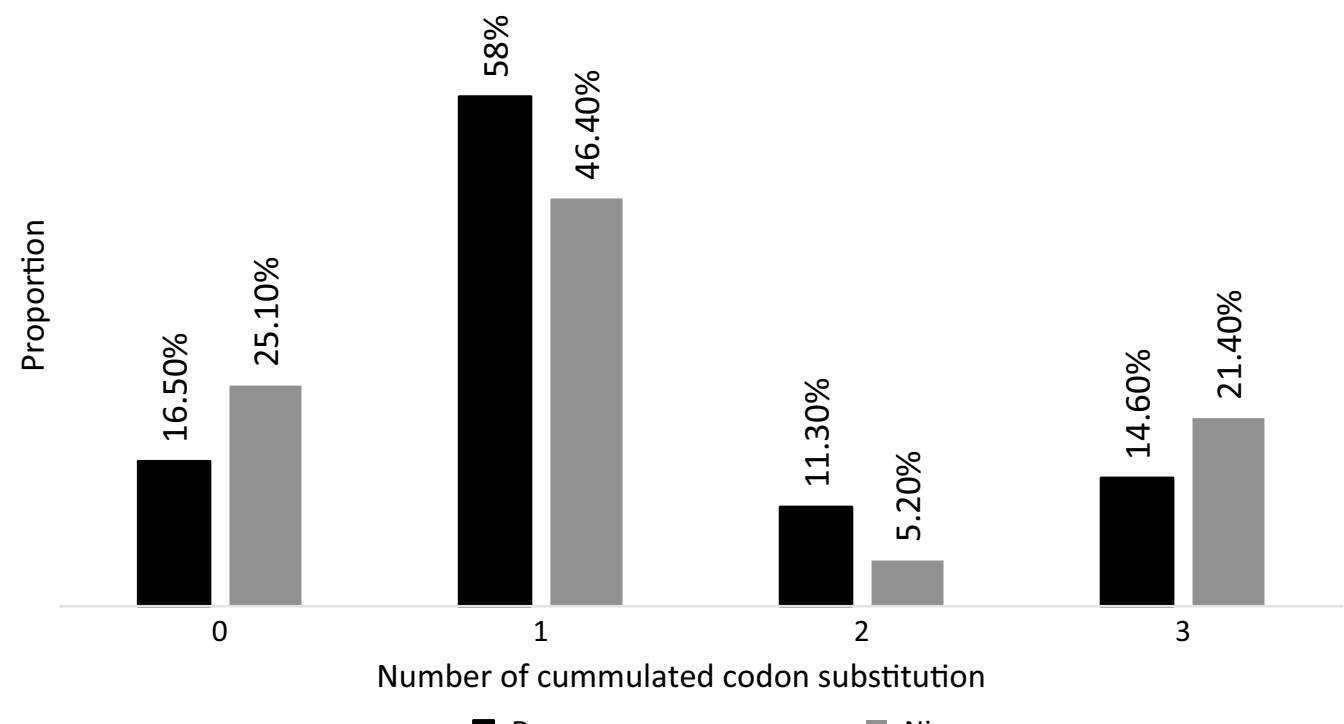

Dangassa

Nioro

Fig. 2 Proportion of number of accumulated codon-substitution in Pfdhfr gene at positions 51, 59, 108 and 164 in Dangassa and Nioro-du-sahel

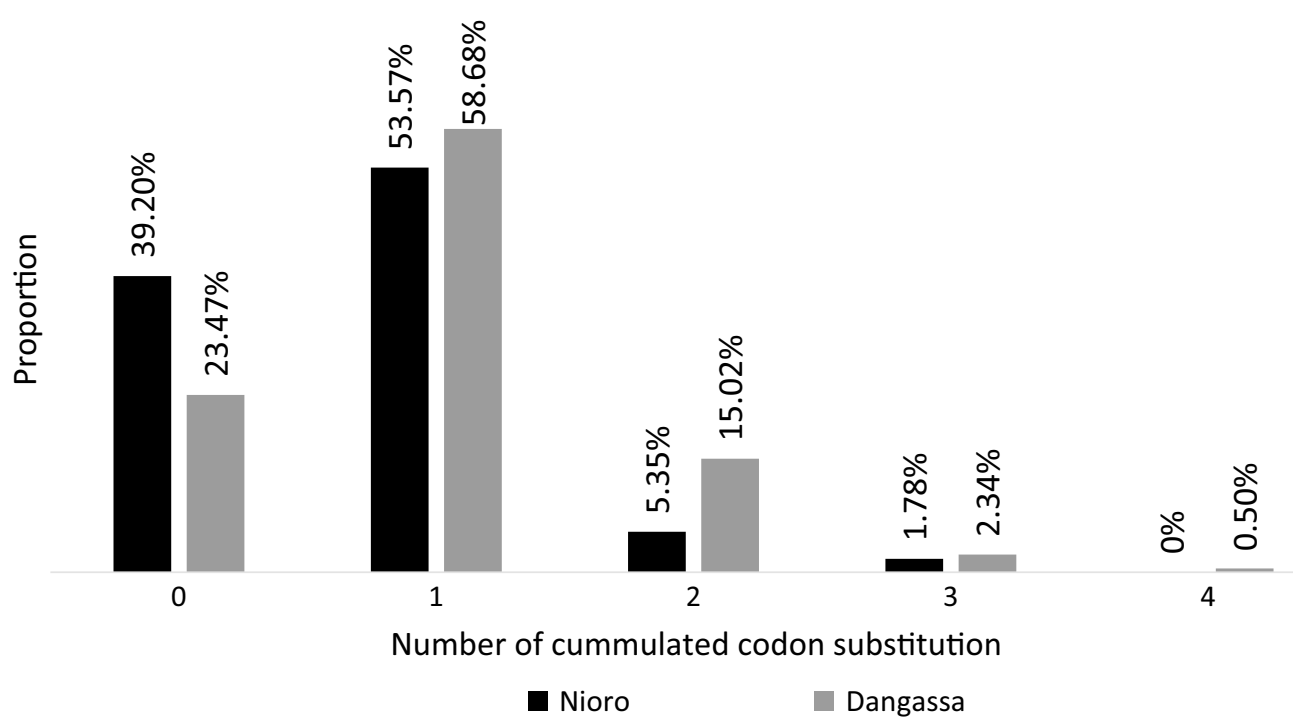

Fig. 3 Proportion of number of accumulated codon-substitution in Pfdhps gene at positions 436, 437A, 540, 581 and 613 in Dangassa and Nioro-du-sahel

More importantly, the lumefantrine-reduced susceptibility Pfmdr1_184F mutation was found in $39.9 \%$ and $48.2 \%$ of samples from Dangassa and Nioro-du-Sahel, respectively. Only two (2) isolates from Dangassa exhibited the piperaquine-resistance Exo-E415G mutation. Such mutation was not found in Nioro. No artemisininsresistance PGB was detected in the genotyped isolates. However, isolated codon substitutions constitutive of the artemisinin resistance PGB were found. Further information regarding the codon variation in studied genes is available in Additional file 1: Table S1.

A principal component analysis of genotyping data from the Sanger 101 SNPs barcode showed very high $P$. falciparum genomic diversity in both Dangassa and Nioro-du-Sahel. However, no aggregation of specific $P$. falciparum genotype was found either in Dangassa or in Nioro (Fig. 4). Higher proportion of multiplicity of infection was observed in Dangassa using either COIL 


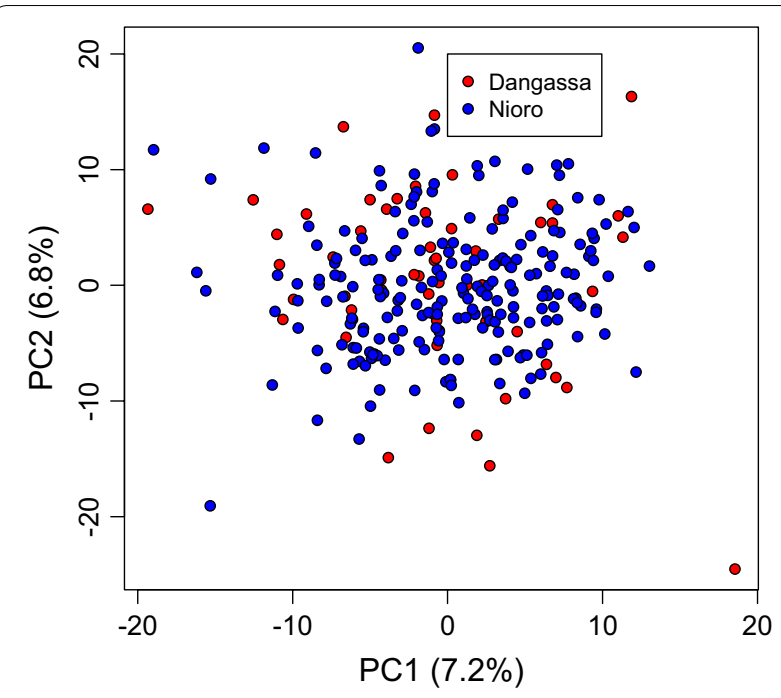

Fig. 4 Plot Visualization of dot from Principal Component (PC) analysis of the 101 SNPs constitutive of barcode. This plot shows a wide distribution of the samples from both Dangassa and Nioro-du-sahel, with no aggregation of the dots

Table 1 Multiplicity of infection in Dangassa and Niorodu-Sahel patients, either with the MOI-COIL or the MOIMcCOIL

\begin{tabular}{llll}
\hline $\begin{array}{l}\text { Index } \\
\text { of multiplicity } \\
\text { of infection }\end{array}$ & $\begin{array}{l}\text { Dangassa } \\
(n=214)\end{array}$ & $\begin{array}{l}\text { Nioro-du- } \\
\text { sahel }(n=56)\end{array}$ & Difference $p$ \\
\hline
\end{tabular}

\begin{tabular}{lrrrrr}
\hline MOI-COIL & & & & & \\
1 & 139 & 65.3 & 44 & 83.0 & \\
2 & 73 & 34.3 & 9 & 17.0 & \\
3 & 1 & 0.5 & 0 & 0.0 & 0.043 \\
MOI-McCOIL & & & & & \\
1 & 151 & 70.9 & 47 & 88.7 & \\
2 & 61 & 28.6 & 6 & 11.3 & \\
3 & 1 & 0.5 & 0 & 0.0 & 0.029 \\
\hline
\end{tabular}

$(\mathrm{p}=0.04)$ or Real McCOIL $(\mathrm{p}=0.02)$ method based on the Sanger 101 SNPs barcode (Table 1).

\section{Discussion}

To protect the artemisinin molecules from resistance emergence in Africa and delay the spread of this resistance in the world, the WHO recommended ACT for uncomplicated malaria treatment in 2001 [4]. Such a strategy requires monitoring $P$. falciparum resistance to anti-malarial drugs in different geographical areas and adapting the appropriate drug combination. Moreover, the monitoring of the malaria parasite genetic variation will help to predict the dynamic of spread of the drug resistance through varying geographical areas. To assess molecular markers of anti-malarial drugs resistance and the genetic diversity in $P$. falciparum circulating isolates through the Malian territory, a passive case detection study was carried out in Dangassa and Nioro, two distinct epidemiological and eco-climatic zones in Mali. The revealed a high prevalence of the chloroquine resistance Pfcrt_76T in both localities. The Pfcrt_76T mutation was found in $64.3 \%$ in Dangassa and $42.5 \%$ in Nioro-du-Sahel. This is the first data report of anti-malarial drug resistance marker in Nioro, but a previous study has reported the prevalence of the chloroquine-resistance Pfcrt_76T mutation was of $85 \%$ in 2002 and $64.5 \%$ in 2003 in Kollé, a village sharing the same geographical area with Dangassa [31]. These observations suggest no decrease in the prevalence of chloroquine resistance marker in this region from 2002 to 2016 despite the official removal of chloroquine by the Malian NMCP since the years 2000s. Several studies in countries where chloroquine was removed reported substantial decrease in the prevalence of the chloroquine resistance marker suggesting a possible future reintroduction of this low-cost drug [43-46].

The data from this study revealed a very low prevalence of amodiaquine resistance-associated mutation Pfmdr1_86Y mutant parasite (two (2/214) in Dangassa and one (1/56) in Nioro-du-Sahel). The Pfmdr1_86Y mutation has been associated with amodiaquine resistance in Burkina Faso [47]. The Pfmdr_86Y mutant isolates was reported at the prevalence of $49.3 \%$ in Kolle, in 2002; however, the authors could not find association between this mutation and amodiaquine resistance in an in vivo assay [31]. Amodiaquine is one of most used antimalarial drugs in Mali; it is associated with artemisinin in ACT and is also used in association with the SP in seasonal malaria control strategy $(\mathrm{SMC})$ in children under five (5) years old in Mali.

Surprisingly, a high prevalence of Pfmdr1_Y184F, the lumefantrine-reduced susceptibility mutation was found in both Dangassa (39.9\%) and Nioro-du-Sahel (48.2\%). A higher prevalence of this mutation (53.8\%) was reported in Senegal in 2013-2014 [48]. This finding was intriguing since lumefantrine has never been made available as a monotherapy in Mali. Lumefantrine has always been administrated in combination with artemether. Two possible scenarios may explain this finding. First, P. falciparum is less susceptible to the lumefantrine. This could impede on the efficacy of artemisinin-based strategy since artemether-lumefantrine is the most used ACT in Mali. Second, the Pfmdr1_Y184F mutation is not a good lumefantrine-resistance marker in Mali. Although several studies have reported an association between Pfmdr1 Y184F mutation and ex vivo reduced susceptibility to 
lumefantrine $[49,50]$, another in vivo studies could not establish this association [51]. Further in vitro investigations are needed to tease this observation apart.

The codon substitutions were assessed in the Pfdhfr and Pfdhp genes to identify SP resistant parasites, characterized by the inheritance of the Pfdhfr_51R-59N108I/Pfdhps_437G-540E quintuple mutation. A high level of codon substitution was observed in these genes. The pyrimethamine-resistance Pfdhfr_51R-59N-108I genotype was found in $14.1 \%$ in Dangassa and $19.6 \%$ in Niorodu-Sahel suggesting a decrease in the prevalence of this haplotype in the south of Mali as compared to the prevalence's of $31.5 \%$ and $42.9 \%$ reported respectively 2002 and 2003 in Kolle [31]. The sulfadoxine resistance haplotype Pfdhps_437G-540E was found in only four isolates in Dangassa. Only one isolate presented the Pfdhfr_51R59N-108I/Pfdhps_437G-540E quintuple substitution in Dangassa. SP is widely used in Mali for pregnant woman malaria chemoprophylaxis and seasonal malaria chemoprophylaxis in children under 5 years old. The data from this study suggest that the use of SP is still relevant in Mali, but previous reports suggest that such a malaria control strategy appears to increase the prevalence of SPresistant parasite in Mali [32]. A very high rate of codon substitution was observed in these two genes (Pfdhfr and Pfdhps) in both Dangassa and Nioro-du-Sahel (Additional file 2).

No Pfarps10_127M-128Y/H-Pffd_193Y-Pfcrt_326S356T-Pfmdr2_484I artemisinin resistance genetic background genotype was found during this study. Several mutations in P. falciparum kelch 13 protein propeller domain have been associated to resistance to artemisinins in Asia [28-30]. A genome wide association study revealed that these mutations always arise on the above specific genetic background [27]. Even though, no artemisinin resistance has been reported yet in Mali, $[5,52]$, a close monitoring for artemisinin resistance is essential. Further analysis of individual mutation constitutive of artemisinin resistance genetic background genotype may provide appropriate tool to predict artemisinin resistance emergence in Africa, where mutation in the Pfkelch13 propeller domain were not associated with artemisinin resistance [53].

P. falciparum genetic diversity is correlated with the level of transmission in different continental regions [54-56], and is influenced within regions by factors such as altitude, vector availability, urbanization, and malaria control strategies $[57,58]$. In this study, it was hypothesized that the eco-climatic difference determines the malaria transmission variability between the two sites, and would impact the fitness of malaria parasites. Therefore, specific genetic variant of parasites could be selected in each site. Using the Sanger's P. falciparum barcodes formed by concatenated genotypes at 101 SNPs across P. falciparum genome, the genetic diversity in P. falciparum circulating isolates was assessed in the study localities. These genotyping data showed a very high genetic diversity in P. falciparum isolates in both Dangassa and Nioro-du-Sahel supporting previous finding in Africa, even though those studies explored the msp1, msp2 or glurp genes size polymorphisms $[59,60]$. No aggregation of specific P. falciparum genotype was observed at either study site. This finding may be due to the fact that the two sites are not geographically isolated. A higher complexity of P. falciparum infection was found in Dangassa, compared to Nioro-du-Sahel. These findings reflect the difference of transmission intensity between the two study sites. The complexity of infection is known to be associated with the intensity of malaria transmission [61, 62].

\section{Conclusions}

This study revealed high diversity in P. falciparum circulating isolates in Mali. However no genetic population structure was found in either low or high transmission areas. No artemisinin resistance genetic background was found, but high prevalence of the lumefantrine resistance marker (Pfmdr1_Y184F genotype), the most used associated molecule to artemisinin in Mali, was detected. Further investigations are required to explore the lumefantrine resistance in Mali.

\section{Supplementary information}

Supplementary information accompanies this paper at https://doi. org/10.1186/s12936-019-2986-5.

Additional file 1: Table S1. Baseline characteristics of the participants. Table S2. Clinical characteristics of participants.

Additional file 2: Codon substitutions in drug resistance genes.

\section{Abbreviations}

USTTB: University of Sciences, Technics and Technologies of Bamako; WACCBIP: West African Centre of Cell Biology of Infectious Pathogens; NGS: nextgeneration sequencing; PfCRT: Plasmodium falciparum chloroquine resistance transporter; PfDHFR: Plasmodium falciparum dihydrofolate-reductase; PfDHPS: Plasmodium falciparum dihydropteroate-synthetase; PfARPS10: Plasmodium falciparum Apicoplast Ribosomal Protein S10; PfFd: Plasmodium falciparum Ferredoxin; PfExo: Plasmodium falciparum exonuclease; PfMDR: Plamodium falciparum Multidrug resistance; COIL: complexity of infection likelihood; McCOIL: Method for the concurrent estimation of complexity of infection likelihood; NMCP: National Malaria Control Programme; ACT: artemisininbased combination therapy; Artemisinins: artemisinin and its derivative drugs; WHO: World Health Organization; SNP: single nucleotid polymorphism; GWAS: Genome-Wide Association Study; PGB: parasite genetic background; LLINs: long lasting insecticide-impregnated nets; IPTp: intermittent preventive treatment of pregnant women; SP: sulfadoxine + pyrimethamine; RDT: rapid diagnostic test; HRP2: histidine rich-protein 2; pLDH: Plasmodium lactate dehydrogenage; i.m: intra muscular; WAG: whole genome amplification.

\section{Acknowledgements}

We thank the parents, guardians and children who participated into this study, and the technical, clinical and nursing staff for assistance. We are grateful to many colleagues at MRTC for providing critical reviews of the manuscripts 
which helped improve it. This publication uses data from the MalariaGEN SpotMalaria Project as described online https://www.malariagen.net/projects/ spotmalaria pending citeable publication; the project is coordinated by the MalariaGEN Resource Centre with funding from Wellcome $(206194,090770)$. The authors would like to thank the staff of Wellcome Sanger Institute Sample Management, Genotyping, Sequencing and Informatics teams for their contribution".

\section{Authors' contributions}

Study setup; SASD; MD Sample collection, data collection: SASD; IS; SK; KT; AC; FD; MAG; MT; MD. Data analysis: SASD; SC; TGC; Manuscript writing: SASD Manuscript review: SASD; DD; AD; NS; SC, MS; GAA; TGC, SD; MD. All authors read and approved the final manuscript.

\section{Funding}

Diakite SAS is supported by a DELTAS Africa grant (DEL-15-007: Awandare). The DELTAS Africa Initiative is an independent funding scheme of the African Academy of Sciences (AAS)'s Alliance for Accelerating Excellence in Science in Africa (AESA) and supported by the New Partnership for Africa's Development Planning and Coordinating Agency (NEPAD Agency) with funding from the Wellcome Trust (107755/Z/15/Z: Awandare) and West Africa ICEMR (2). Genotyping was done at the Sanger Institute thanks to the Wellcome Trust Sanger Institute Grant No. 098051 to DK. TGC is funded by the Medical Research Council UK (Grant No. MR/M01360X/1, MR/N010469/1, MR/R025576/1, and MR/R020973/1) and BBSRC (Grant No. BB/R013063/1). SC is funded by Medical Research Council UK Grants (MR/M01360X/1, MR/R025576/1, and MR/ R020973/1) and BBSRC (Grant No. BB/R013063/1).

\section{Availability of data and materials}

Public accession numbers for raw sequence data analysed are contained in SRA studies ERP000190 and ERP000199, as well as being accessible from the Pf3k project website (https://www.malariagen.net/projects/pf3k).

\section{Ethics approval and consent to participate}

Before starting this study, we obtained a community consent from traditional and customary chiefs prior to the study. The study was approved by the ethics committee of the faculty of medicine and Pharmacy of the University of Sciences, Technics and Technologies of Bamako (USTTB), Mali. Written informed consent was obtained from a parent or guardian of each enrolled child.

\section{Consent for publications}

All authors read and approved the final manuscript.

\section{Competing interests}

The authors declare that they have no competing interests.

\author{
Author details \\ ${ }^{1}$ Malaria Research and Training Center, University of Sciences, Technics \\ and Technologies of Bamako (USTTB), Bamako, Mali. ${ }^{2}$ West African Centre \\ for Cell Biology of Infectious Pathogens, University of Ghana, Accra, Ghana. \\ ${ }^{3}$ Faculty of Infectious and Tropical Diseases, London School of Hygiene \\ and Tropical Medicine, London, UK.
}

Received: 6 May 2019 Accepted: 24 October 2019

Published online: 12 November 2019

\section{References}

1. WHO. World malaria report 2018. Geneva: World Health Organization; 2018.

2. Bloland PB. Drug resistance in malaria. Geneva: World Health Organization, WHO/CDS/CSR/DRS; 2001.

3. Sinha $S$, Medhi B, Sehgal R. Challenges of drug-resistant malaria. Parasite. 2014;21:61.

4. WHO. Antimalarial drug combination therapy. Geneva: World Health Organization; 2001.

5. Lopera-Mesa TM, Doumbia S, Chiang S, Zeituni AE, Konate DS, Doumbouya $\mathrm{M}$, et al. Plasmodium falciparum clearance rates in response to artesunate in Malian children with malaria: effect of acquired immunity. J Infect Dis. 2013;207:1655-63.
6. Abdulla S, Ashley EA, Bassat Q, Bethell D, Bjorkman A, Borrmann S, et al. Baseline data of parasite clearance in patients with falciparum malaria treated with an artemisinin derivative: an individual patient data metaanalysis. Malar J. 2015;14:359.

7. Dondorp AM, Fairhurst RM, Slutsker L, Macarthur JR, Breman JG, Guerin PJ, et al. The threat of artemisinin-resistant malaria. N Engl J Med. 2011;365:1073-5.

8. Enserink M. Malaria's drug miracle in danger. Science. 2010;328:844-6.

9. WHO. Global plan for artemisinin resistance containment (GPARC). Geneva: World Health Organization; 2012.

10. Hastings IM. Molecular markers as indicators of antimalarial drug failure rates. Trop Med Int Health. 2007;12:1298-301.

11. Foote SJ, Kyle DE, Martin RK, Oduola AM, Forsyth K, Kemp DJ, et al. Several alleles of the multidrug-resistance gene are closely linked to chloroquine resistance in Plasmodium falciparum. Nature. 1990;345:255-8.

12. Fidock DA, Nomura T, Talley AK, Cooper RA, Dzekunov SM, Ferdig MT, et al. Mutations in the $\mathrm{P}$ falciparum digestive vacuole transmembrane protein PfCRT and evidence for their role in chloroquine resistance. Mol Cell. 2000;6:861-71.

13. Baro NK, Callaghan PS, Roepe PD. Function of resistance conferring Plasmodium falciparum chloroquine resistance transporter isoforms. Biochemistry. 2013;52:4242-9.

14. Ecker A, Lehane AM, Clain J, Fidock DA. PfCRT and its role in antimalarial drug resistance. Trends Parasitol. 2012;28:504-14.

15. Durand R, Jafari S, Vauzelle J, Delabre JF, Jesic Z, Le Bras J. Analysis of pfcrt point mutations and chloroquine susceptibility in isolates of Plasmodium falciparum. Mol Biochem Parasitol. 2001;114:95-102.

16. Chusacultanachai S, Thiensathit P, Tarnchompoo B, Sirawaraporn W, Yuthavong Y. Novel antifolate resistant mutations of Plasmodium falciparum dihydrofolate reductase selected in Escherichia coli. Mol Biochem Parasitol. 2002;120:61-72.

17. Gregson A, Plowe CV. Mechanisms of resistance of malaria parasites to antifolates. Pharmacol Rev. 2005;57:117-45.

18. Peterson DS, Walliker D, Wellems TE. Evidence that a point mutation in dihydrofolate reductase-thymidylate synthase confers resistance to pyrimethamine in falciparum malaria. Proc Natl Acad Sci USA. 1988;85:9114-8.

19. Wang P, Lee CS, Bayoumi R, Djimde A, Doumbo O, Swedberg G, et al. Resistance to antifolates in Plasmodium falciparum monitored by sequence analysis of dihydropteroate synthetase and dihydrofolate reductase alleles in a large number of field samples of diverse origins. Mol Biochem Parasitol. 1997;89:161-77.

20. Nsobya SL, Kiggundu M, Nanyunja S, Joloba M, Greenhouse B, Rosenthal PJ. In vitro sensitivities of Plasmodium falciparum to different antimalarial drugs in Uganda. Antimicrob Agents Chemother. 2010;54:1200-6.

21. Echeverry DF, Holmgren G, Murillo C, Higuita JC, Bjorkman A, Gil JP, et al. Polymorphisms in the pfcrt and pfmdr1 genes of Plasmodium falciparum and in vitro susceptibility to amodiaquine and desethylamodiaquine. Am J Trop Med Hyg. 2007;77:1034-8.

22. Sidhu $A B$, Valderramos SG, Fidock DA. Pfmdr1 mutations contribute to quinine resistance and enhance mefloquine and artemisinin sensitivity in Plasmodium falciparum. Mol Microbiol. 2005;57:913-26.

23. Malmberg M, Ferreira PE, Tarning J, Ursing J, Ngasala B, Bjorkman A, et al. Plasmodium falciparum drug resistance phenotype as assessed by patient antimalarial drug levels and its association with pfmdr1 polymorphisms. J Infect Dis. 2013;207:842-7.

24. Taylor AR, Flegg JA, Holmes CC, Guerin PJ, Sibley CH, Conrad MD, et al. Artemether-lumefantrine and dihydroartemisinin-piperaquine exert inverse selective pressure on Plasmodium falciparum drug sensitivityassociated haplotypes in Uganda. Open Forum Infect Dis. 2017;4:229.

25. Sisowath C, Ferreira PE, Bustamante LY, Dahlstrom S, Martensson A, Bjorkman $A$, et al. The role of pfmdr 1 in Plasmodium falciparum tolerance to artemether-lumefantrine in Africa. Trop Med Int Health. 2007;12:736-42.

26. Amato R, Lim P, Miotto O, Amaratunga C, Dek D, Pearson RD, et al. Genetic markers associated with dihydroartemisinin-piperaquine failure in Plasmodium falciparum malaria in Cambodia: a genotype-phenotype association study. Lancet Infect Dis. 2017;17:164-73.

27. Miotto O, Amato R, Ashley EA, Maclnnis B, Almagro-Garcia J, Amaratunga C, et al. Genetic architecture of artemisinin-resistant Plasmodium falciparum. Nat Genet. 2015;47:226-34. 
28. Ashley EA, Dhorda M, Fairhurst RM, Amaratunga C, Lim P, Suon S, et al. Spread of artemisinin resistance in Plasmodium falciparum malaria. N Engl J Med. 2014;371:411-23.

29. Ariey F, Witkowski B, Amaratunga C, Beghain J, Langlois AC, Khim N, et al. A molecular marker of artemisinin-resistant Plasmodium falciparum malaria. Nature. 2014;505:50-5.

30. Tun KM, Imwong M, Lwin KM, Win AA, Hlaing TM, Hlaing T, et al. Spread of artemisinin-resistant Plasmodium falciparum in Myanmar: a cross-sectional survey of the K13 molecular marker. Lancet Infect Dis. 2015;15:415-21.

31. Tekete M, Djimde AA, Beavogui AH, Maiga H, Sagara I, Fofana B, et al. Efficacy of chloroquine, amodiaquine and sulphadoxine-pyrimethamine for the treatment of uncomplicated falciparum malaria: revisiting molecular markers in an area of emerging $\mathrm{AQ}$ and SP resistance in Mali. Malar J. 2009;8:34.

32. Maiga H, Lasry E, Diarra M, Sagara I, Bamadio A, Traore A, et al. Seasonal malaria chemoprevention with sulphadoxine-pyrimethamine and amodiaquine selects Pfdhfr-dhps quintuple mutant genotype in Mali. PLoS One. 2016;11:e0162718.

33. Snow RW, Craig MH, Deichmann U, le Sueur D. A preliminary continental risk map for malaria mortality among African children. Parasitol Today. 1999;15:99-104.

34. Tanser FC, Sharp B, le Sueur D. Potential effect of climate change on malaria transmission in Africa. Lancet. 2003;362:1792-8.

35. Sogoba N, Keita M, Diakite M, Diallo M, Baber I, Manoukis N, et al. Malaria transmission along the Niger river in a Sudan Savanna area of Mali. Am J Trop Med Hyg. 2009;81:S170.

36. WHO. Severe falciparum malaria World Health Organization, Communicable Diseases Cluster. Trans R Soc Trop Med Hyg. 2000;94(Suppl 1):S1-90.

37. Zhang L, Cui X, Schmitt K, Hubert R, Navidi W, Arnheim N. Whole genome amplification from a single cell: implications for genetic analysis. Proc Natl Acad Sci USA. 1992;89:5847-51.

38. Oyola SO, Ariani CV, Hamilton WL, Kekre M, Amenga-Etego LN, Ghansah A, et al. Whole genome sequencing of Plasmodium falciparum from dried blood spots using selective whole genome amplification. Malar J. 2016;15:597.

39. Manske M, Miotto O, Campino S, Auburn S, Almagro-Garcia J, Maslen G, et al. Analysis of Plasmodium falciparum diversity in natural infections by deep sequencing. Nature. 2012;487:375-9.

40. Chang HH, Wesolowski A, Sinha I, Jacob CG, Mahmud A, Uddin D, et al. Mapping imported malaria in Bangladesh using parasite genetic and human mobility data. Elife. 2019;8:e43481.

41. Galinsky K, Valim C, Salmier A, de Thoisy B, Musset L, Legrand E, et al. COIL: a methodology for evaluating malarial complexity of infection using likelihood from single nucleotide polymorphism data. Malar J. 2015;14:4.

42. Chang HH, Worby CJ, Yeka A, Nankabirwa J, Kamya MR, Staedke SG, et al. THE REAL MCCOIL: a method for the concurrent estimation of the complexity of infection and SNP allele frequency for malaria parasites. PLoS Comput Biol. 2017;13:e1005348.

43. Ndam NT, Basco LK, Ngane VF, Ayouba A, Ngolle EM, Deloron P, et al. Reemergence of chloroquine-sensitive pfcrt K76 Plasmodium falciparum genotype in southeastern Cameroon. Malar J. 2017;16:130.

44. Thomsen TT, Madsen LB, Hansson HH, Tomas EV, Charlwood D, Bygbjerg IC, et al. Rapid selection of Plasmodium falciparum chloroquine resistance transporter gene and multidrug resistance gene-1 haplotypes associated with past chloroquine and present artemether-lumefantrine use in Inhambane District, southern Mozambique. Am J Trop Med Hyg. 2013;88:536-41.

45. Laufer MK, Thesing PC, Dzinjalamala FK, Nyirenda OM, Masonga R, Laurens $\mathrm{MB}$, et al. A longitudinal trial comparing chloroquine as monotherapy or in combination with artesunate, azithromycin or atovaquoneproguanil to treat malaria. PLoS One. 2012;7:e42284.

46. Kublin JG, Cortese JF, Njunju EM, Mukadam RA, Wirima JJ, Kazembe PN, et al. Reemergence of chloroquine-sensitive Plasmodium falciparum malaria after cessation of chloroquine use in Malawi. J Infect Dis. 2003;187:1870-5
47. Dokomajilar C, Lankoande ZM, Dorsey G, Zongo I, Ouedraogo JB, Rosenthal PJ. Roles of specific Plasmodium falciparum mutations in resistance to amodiaquine and sulfadoxine-pyrimethamine in Burkina Faso. Am J Trop Med Hyg. 2006;75:162-5.

48. Boussaroque A, Fall B, Madamet M, Wade KA, Fall M, Nakoulima A, et al. Prevalence of anti-malarial resistance genes in Dakar, Senegal from, to 2014. Malar J. 2013;15:347.

49. Wurtz N, Fall B, Pascual A, Fall M, Baret E, Camara C, et al. Role of Pfmdr1 in in vitro Plasmodium falciparum susceptibility to chloroquine, quinine, monodesethylamodiaquine, mefloquine, lumefantrine, and dihydroartemisinin. Antimicrob Agents Chemother. 2014;58:7032-40.

50. Dama S, Niangaly H, Ouattara A, Sagara I, Sissoko S, Traore OB, et al. Reduced ex vivo susceptibility of Plasmodium falciparum after oral artemether-lumefantrine treatment in Mali. Malar J. 2017;16:59.

51. Baraka V, Mavoko HM, Nabasumba C, Francis F, Lutumba P, Alifrangis M, et al. Impact of treatment and re-treatment with artemether-lumefantrine and artesunate-amodiaquine on selection of Plasmodium falciparum multidrug resistance gene-1 polymorphisms in the Democratic Republic of Congo and Uganda. PLoS One. 2018;13:e0191922.

52. Maiga AW, Fofana B, Sagara I, Dembele D, Dara A, Traore OB, et al. No evidence of delayed parasite clearance after oral artesunate treatment of uncomplicated falciparum malaria in Mali. Am J Trop Med Hyg. 2012;87:23-8

53. WWARN K13 Genotype-Phenotype Study Group. Association of mutations in the Plasmodium falciparum kelch13 gene (Pf3D7_1343700) with parasite clearance rates after artemisinin-based treatments-a WWARN individual patient data meta-analysis. BMC Med. 2019;17:1.

54. Anderson TJ, Haubold B, Williams JT, Estrada-Franco JG, Richardson L, Mollinedo R, et al. Microsatellite markers reveal a spectrum of population structures in the malaria parasite Plasmodium falciparum. Mol Biol Evol. 2000;17:1467-82

55. Mu J, Awadalla P, Duan J, McGee KM, Joy DA, McVean GA, et al. Recombination hotspots and population structure in Plasmodium falciparum. PLoS Biol. 2005;3:e335.

56. Joy DA, Feng X, Mu J, Furuya T, Chotivanich K, Krettli AU, et al. Early origin and recent expansion of Plasmodium falciparum. Science. 2003;300:318-21.

57. Anthony TG, Conway DJ, Cox-Singh J, Matusop A, Ratnam S, Shamsul S, et al. Fragmented population structure of Plasmodium falciparum in a region of declining endemicity. J Infect Dis. 2005;191:1558-64.

58. Tsumori Y, Ndounga M, Sunahara T, Hayashida N, Inoue M, Nakazawa S, et al. Plasmodium falciparum: differential selection of drug resistance alleles in contiguous urban and peri-urban areas of Brazzaville, Republic of Congo. PLoS One. 2011;6:e23430.

59. Babiker HA, Lines J, Hill WG, Walliker D. Population structure of Plasmodium falciparum in villages with different malaria endemicity in east Africa. Am J Trop Med Hyg. 1997;56:141-7.

60. Ghanchi NK, Martensson A, Ursing J, Jafri S, Bereczky S, Hussain R, et al. Genetic diversity among Plasmodium falciparum field isolates in Pakistan measured with PCR genotyping of the merozoite surface protein 1 and 2 . Malar J. 2010;9:1

61. Basco LK, Ringwald P. Molecular epidemiology of malaria in Yaounde, Cameroon. VII. Analysis of recrudescence and reinfection in patients with uncomplicated falciparum malaria. Am J Trop Med Hyg. 2000;63:215-21.

62. Happi CT, Gbotosho GO, Sowunmi A, Falade CO, Akinboye DO, Gerena L, et al. Molecular analysis of Plasmodium falciparum recrudescent malaria infections in children treated with chloroquine in Nigeria. Am J Trop Med Hyg. 2004;70:20-6.

\section{Publisher's Note}

Springer Nature remains neutral with regard to jurisdictional claims in published maps and institutional affiliations. 